\title{
Estudos florísticos no município de Presidente Figueiredo, Amazonas. Brasil - I. Famílias Annonaceae e Gnetaceae
}

\author{
José Augusto Coelho da SILVA ${ }^{1}$, Marlene Freitas da SILVA²,3
}

\begin{abstract}
RESUMO
O Município de Presidente Figueiredo, no Estado do Amazonas, foi visitado durante três anos consecutivos, de 1996 a 1999, com o objetivo de se obter amostras botânicas da área, para estudos no Subprojeto "Elaboração de Revisões e Monografias Taxonômicas" com a finalidade de se elaborar uma Flórula para o Município. As coleções feitas neste período encontram-se depositadas no acervo do Herbário do INPA e as informações gerais sobre os taxa aqui estudados foram complementadas com as obtidas das coleções feitas anteriormente por outros pesquisadores. Para a família Annonaceae Juss. (Magnoliopsida) foram identificadas 39 espécies distribuídas em 15 gêneros. Destes, Duguetia A. St. Hill., Guatteria Ruiz \& Pavon, Annona L., Unonopsis R.E.Fries e Xylopia L. destacaram-se pela diversidade em espécies, sendo os dois primeiros representados por oito e seis espécies, respectivamente, e os três últimos, com quatro espécies. A família Gnetaceae Lindl. (Gimnospermae) está representada no Município apenas pelo gênero Gnetum L., com a espécie G. leyboldii Tul.
\end{abstract}

PALAVRAS-CHAVE

Presidente Figueiredo, Lista de espécies, Annonaceae, Gnetaceae.

\section{Floristic studies in township of Presidente Figueiredo, Amazonas. Brazil - I. Families Annonaceae and Gnetaceae}

\begin{abstract}
The Township of Presidente Figueiredo, in the State of Amazonas, was visited for three consecutive years, from 1996 to 1999, with the aim of acquiring botanical samples from the area, in order to carry out studies in the "Taxonomic Monographies and Revisions Preparation" SubProject, for the purpose of preparing a survey of the flora. Collections pertaining to the present work are deposited at INPA's herbarium and, general information on the taxa studied here were complemented with those obtained from earlier collections conducted by other researchers. Thirty nine (39) species distributed into 15 genera were identified for the family Annonaceae Juss. (Magnoliopsida). Of these, Duguetia A. St. Hill., Guatteria Ruiz \& Pavon, Annona L., Unonopsis R.E.Fries and Xylopia L. stood out on account of their species diversity, the former two were represented by eight and six species respectively, and the latter three by four species each. Family Gnetaceae Lindl. (Gimnospermae) is only represented in the surveyed township by genus Gnetum L., with species G. leyboldii Tul.
\end{abstract}

\section{KEYWORDS}

Presidente Figueiredo, Check list, Annonaceae, Gnetaceae.

\footnotetext{
${ }^{1}$ Instituto Nacional de Pesquisas da Amazônia (INPA), Coordenação de Pesquisas em Botânica, Caixa Postal 478, CEP 69.060-001-Manaus AM. E-mail : augusto@inpa.govbr ${ }^{2}$ Universidade do Estado do Amazonas (UEA.), Coordenadora do Curso de Pós-Graduação em Biotecnologia de Produtos Naturais da Amazônia e Professora Titular do Curso de Engenharia Florestal da UEA. Instituto de Tecnologia da Amazônia (UTAM), Av. Darcy Vargas, 1200, CEP 69.050-020.

${ }^{3}$ In memorian
} 


\section{INTRODUÇÃO}

O Município de Presidente Figueiredo está situado á nordeste do Estado do Amazonas, norte de Manaus, e é delimitado pela linha do Equador, paralelo $3^{\circ} 00^{\prime} 00^{\prime \prime}$ e pelos meridianos 61030'00" e 58 30'00”, perfazendo uma área de $24.781 \mathrm{Km}^{2}$. (Fig. 1).

A cobertura vegetal do Município é constituída, principalmente, por floresta ombrófila densa de terra firme e os principais acidentes geográficos são: cachoeiras, corredeiras, cavernas, igarapés, lagos e rios que, devido ás inundações periódicas, durante a estação chuvosa, mantêm em suas margens a floresta de igapó.

Embora a região tenha sofrido uma grande exploração seletiva do pau-rosa (Aniba rosaeodora Ducke, Lauraceae), a vegetação do Município é ainda em grande parte constituída por florestas naturais, com poucas áreas perturbadas pela ação antrópica (Eletronorte/Ibama,1996).

A família Annonaceae é representada nos Neotrópicos por cerca de 40 gêneros e aproximadamente 650 espécies, com centro de distribuição na região amazônica e Guianas. (Ribeiro, et al. 1999).

Caracterizada por apresentar quase que exclusivamente hábito arborescente, arvoretas ou, raramente, na forma de lianas (Annona baematantha Miq.). Vegetativamente, a família pode ser reconhecida pelas seguintes características particulares: odor forte e característico que exala do tronco cortado ou dos ramos; por apresentar fibras longas e resistentes na casca, conhecidas popularmente como envira; pela filotaxia alterna $\mathrm{e}$ dística de suas folhas (exceto em Tetramerantbus R.E.Fries, com folhas espiraladas) e, pela presença de marcas de chamas no corte transversal do tronco. Pêlos ou escamas estreladas são indumentos típicos do gênero Duguetia A.St-Hill. e ocorrem, também, em Tetrameranthus (Ribeiro, et al. 1999).

A maioria das espécies de Annonaceae está quase que totalmente limitada aos trópicos, onde crescem em baixas altitudes, e o maior número de gêneros ocorre nas Américas ( \pm 40 gêneros), com predominância na América do Sul ( \pm 35 gêneros). O grande centro de distribuição da família é, sem dúvida, a região amazônica, as Guianas e o sudeste do Brasil. Suas espécies habitam as florestas de terra firme, as várzeas, os cerrados e os campos (Miralha, 1995).

A família Gnetaceae, com um único gênero, Gnetum L., e aproximadamente 35 espécies, encontra-se distribuída nas regiões de clima tropical úmido. $\mathrm{Na}$ Ásia ela está representada por cerca de 25 espécies, na África Ocidental por duas espécies e nos Neotrópicos por cerca de oito a dez espécies, das quais cerca de seis espécies são encontradas na Amazônia. (Cavalcante, 1978 e Ribeiro, et al. 1999).

As espécies americanas mostram mais afinidades com as espécies africanas, o que é explicado, segundo Markgraf (1930), como uma decorrência da teoria da deriva continental.

Além disso, as espécies americanas são todas de hábito lianescente, lenhosas ou herbáceas e, vegetativamente, mais parecidas às dicotiledôneas que as outras gimnospermas. As folhas são simples, opostas, pecioladas, com venação peninérvea e sem estípulas.

O gênero Gnetum pode ser facilmente reconhecido pela combinação dos caracteres acima citados com os nós e entrenós bem distintos nos ramos terminais, parecendo articulações. Algumas espécies apresentam seiva pegajosa no tronco e nos ramos. (Ribeiro, et al. 1999).

Segundo Cavalcante, (1978) as Gnetáceas habitam as áreas de baixa altitude cobertas de florestas, porém ocorrem também nas savanas, campinas ou capoeiras. Em geral não são encontradas no interior da floresta de terra firme, mas, nos limites desta com os rios, furos, canais e igarapés da região.

As espécies amazônicas de Gnetum são de um modo geral conhecidas por "ituá", por seus frutos comestíveis, muito apreciados pela população interiorana. O pseudofruto ou semente é assado e a amêndoa é comestível ou então, pode também ser transformada em "farinha de ituá" para o consumo. O caule é rico em fibras, sendo utilizado para fabricação de cordas bastante resistentes.

Este trabalho visa obter amostras botânicas da área de Presidente Figueiredo, para estudos no Subprojeto "Elaboração de Revisões e Monografias Taxonômicas" com a finalidade de se elaborar um estudo florístico para o Município, e assim contribuir com o turismo ecológico, identificando as espécies vegetais ali existentes, bem como a ocorrência dessas espécies, por intermédio das coleções depositadas no acervo do Herbário do INPA.

\section{ÁREA DE ESTUDO/SOLO/CLIMA}

Presidente Figueiredo é um dos municípios amazonenses de melhor e maior acesso aos visitantes de todo o Estado e que, pela exuberância de seus recursos naturais, apresenta grande potencial para o turismo ecológico, por incorporar em seus limites, o lago da Hidrelétrica de Balbina, propício à prática da pesca desportiva, além de outras atividades turísticas como,

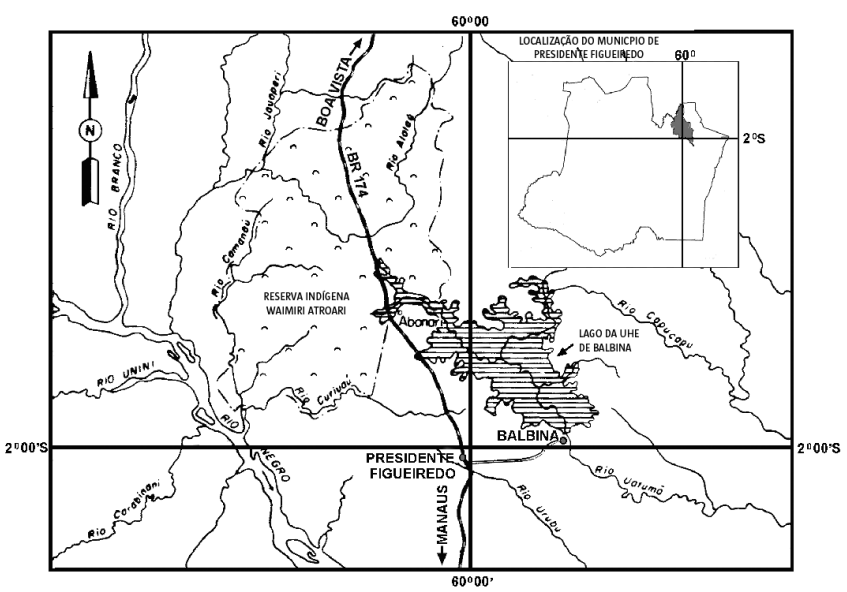

Figura 1 - Mapa de Situação do Município de Presidente Figueiredo (AM). Escala aproximada 1:2.000.000. (Nava et al.1998). 


\section{ACTA \\ AMAZONICA}

ESTUDOS FLORÍSTICOS NO MUNICÍPIO DE PRESIDENTE FIGUEIREDO,

AMAZONAS. BRASIL - I. FAMÍLIAS ANNONACEAE E GNETACEAE

Tabela 1 - Famílias e Espécies encontradas, Nome e Números dos coletores e Freqüência de coleta, no Município de Presidente Figueiredo(AM): Annonaceae e Gnetaceae.

\begin{tabular}{|c|c|c|}
\hline Famílias/Espécies & $\begin{array}{l}N^{\circ} \text { de } \\
\text { Ocorrência }\end{array}$ & Nome e $\mathrm{N}^{\circ}$ de Coletores \\
\hline \multicolumn{3}{|l|}{ I. Annonaceae } \\
\hline 1.1 Anaxagorea brevipes Benth. & 6 & $\begin{array}{l}\text { Berg, C.C. et al. P18135; Cid, C. A. et al. 38, 8094; Dionisía, F. et al. } \\
\text { 198; Lima, J. 565; Loureiro, A. et al. s.n. (INPA 48002). }\end{array}$ \\
\hline 1.2 Anaxagorea phaeocarpa Mart. & 4 & $\begin{array}{l}\text { Lima, J. 586; Loureiro, A. et al. s.n. (INPA 48041; Prance, G. T. et al. } \\
\text { 24259; Steward, W. C. et al. P20396. }\end{array}$ \\
\hline 1.3 Annona echinata Dunal & 1 & Silva, M. F. et al. 1987. \\
\hline 1.4 Annona foetida Mart. & 3 & $\begin{array}{l}\text { Cid, C. A. et al. 6976; Loureiro, A et al. s.n. (INPA 48057); Prance, G. T. } \\
\text { et al. } 22684 .\end{array}$ \\
\hline 1.5 Annona impressivenia Saff. ex R. E. Fr. & 9 & $\begin{array}{l}\text { Cid, C. A. 7562; Cid, C. A. et al. } 66,70,7005,8102 \text {; Gentry, Al. } 12971 \text {; } \\
\text { Lima, J. 597; Loureiro, A et al. s.n. (INPA 47960); Thomas, W. et al. } \\
5328 .\end{array}$ \\
\hline 1.6 Annona sericea Dunal & 1 & Prance, G. T. et al. 24304. \\
\hline 1.7 Bocageopsis multiflora (Mart.) R. E. Fr. & 2 & Bisby, F. et al. P18089; Prance, G. T. et al. 22714. \\
\hline 1.8 Diclinanona calycina (Diels) R. E. Fr. & 2 & Thomas, W. et al. 5268, 5280 . \\
\hline 1.9 Duguetia asterotricha (Diels) R. E. Fr. & 2 & Cid, C. A. et al. 935; Loureiro, A. et al. s.n. (INPA 48072). \\
\hline 1.10 Duguetia cauliflora R. E. Fr. & 3 & $\begin{array}{l}\text { Carvalho, V. et al. 124; Loureiro, A. et al. s.n. (INPA 48148); Thomas, } \\
\text { W. et al. } 5278 .\end{array}$ \\
\hline 1.11 Duguetia flagellaris Huber & 2 & Prance, G. T. et al. 24242; Steward, W. C. et al. 70. \\
\hline 1.12 Duguetia latifolia R. E. Fr. & 1 & Cid, C. A. et al. 6991. \\
\hline 1.13 Duguetia longicuspis Benth. & 2 & Cid, C. A. et al. 6874; Thomas, W. et al. 5365. \\
\hline 1.14 Duguetia megalocarpa Maas & 1 & Loureiro, A. et al. s.n. (INPA 48046). \\
\hline 1.15 Duguetia riparia Huber & 1 & Lima, J. 584. \\
\hline 1.16 Duguetia stelechanta (Diels) R. E. Fr. & 4 & $\begin{array}{l}\text { Cid, C. A. et al. 976, 6716; Steward, W. C. et al. P20373; Thomas, W. } \\
\text { et al. } 5367 .\end{array}$ \\
\hline 1.17 Duguetia sp. & 1 & Cid, C. A. et al. 6757. \\
\hline 1.18 Ephedranthus amazonicus R. E. Fr. & 2 & Bisby, F. et al. P18068; Silva, J. A et al. 797. \\
\hline 1.19 Fusaea longifolia (Aubl.) Saff. & 7 & $\begin{array}{l}\text { Amaral, I. L. et al. 1732; Cid, C. A. et al. 6978, 8245; Coelho, D. et al. } \\
\text { 874; Lima, J. 583; Silva, J. A et al. 820; Steward, W. C. et al. P20271. }\end{array}$ \\
\hline 1.20 Guatteria dielsiana R. E. Fr. & 1 & Cid, C. A et al. 979. \\
\hline 1.21 Guatteria cf. maypurensis Kunth & 1 & Loureiro, A et al. s.n. (INPA 47978). \\
\hline 1.22 Guatteria megalophylla Diels & 8 & $\begin{array}{l}\text { Cid, C. A. et al. } 6949,6986 \text {; Dionísia, F. et al. 204; Lima, J. } 651 \text {; } \\
\text { Loureiro, A. et al. s.n. (INPA 48053); Prance, G. T. et al. 24320; } \\
\text { Steward, W. C. et al. 62, P20385. }\end{array}$ \\
\hline 1.23 Guatteria meliodora R. E. Fr. & 2 & Cid, C. A. et al. 6586; Loureiro, A et al. s.n. (INPA 47931). \\
\hline 1.24 Guatteria schomburgkiana Mart. & 3 & Cid, C. A. et al. 8097; Lima, J. 588; Silva, J. A. et al. 870. \\
\hline 1.25 Guatteria scytophylla Diels & 2 & Cid, C. A. et al. 6871; Steward, W. C. et al. P20244. \\
\hline 1.26 Guatteria sp. & 13 & $\begin{array}{l}\text { Amaral, I. L. et al. 2070; Berg, C. C. et al. P18155; Cid, C. A. et al. } \\
\text { 6593, 6609, 6664, 6973, 6989, 7008, 7587, 8155, 8247; Steward, W. C. } \\
\text { et al. P20269; Thomas, W. et al. 5352. }\end{array}$ \\
\hline $\begin{array}{l}1.27 \text { Guatteriopsis blepharophylla (Mart.) R. } \\
\text { E. Fr. }\end{array}$ & 5 & $\begin{array}{l}\text { Carvalho, V. et al. 129; Cid, C. A. et al. 40, 8203; Miranda, A. P. et al. } \\
\text { s.n. (INPA 48063); Steward, W. C. et al. P19680. }\end{array}$ \\
\hline 1.28 Oxandra xilopioides Diels & 3 & Cid, C. A. et al. 8071; Lopes, M. et al. 127; Prance, G. T. et al. 22718 \\
\hline 1.29 Oxandra sp. & 1 & Cid, C. A. et al. 8090. \\
\hline 1.30 Pseudoxandra coriacea R. E. Fr. & 6 & $\begin{array}{l}\text { Carvalho, V. et al. } 120 \text {; Cid, C. A. } 7576 \text {; Cid, C. A. et al. } 30,6934 \text {, } \\
\text { 8151; Thomas, W. et al. } 5433 \text {. }\end{array}$ \\
\hline 1.31 Pseudoxandra polyphleba R. E. Fr. & 1 & Dionísia, F. et al. 191. \\
\hline 1.32 Rollinia edulis Tr. \& Pl. & 1 & Amaral, I. L. et al. 2077. \\
\hline 1.33 Rollinia exsucca (DC. ex Dunal) A. DC. & 2 & Berg, C. C. et al. P19551; Prance, G. T. et al. 24326. \\
\hline
\end{tabular}


Tabela 1 - Continuação

\begin{tabular}{|c|c|c|}
\hline Famílias/Espécies & $\begin{array}{l}N^{\circ} \text { de } \\
\text { Ocorrência }\end{array}$ & Nome e $N^{\circ}$ de Coletores \\
\hline 1.34 Tetrameranthus laomae D. Simpson & 6 & $\begin{array}{l}\text { Amaral, I. L. et al. } 1751 \text {; Cid, C. A. et al. } 6657,6738,8084 \text {; Thomas, W. } \\
\text { et al. 5271, 5358. }\end{array}$ \\
\hline 1.35 Unonopsis duckei R. E. Fr. & 1 & Carvalho, v. et al. 121. \\
\hline $\begin{array}{l}\text { 1.36 Unonopsis guatterioides (A. DC.) R. E. } \\
\text { Fr. }\end{array}$ & 3 & Cid, C. A. et al. 6914; Lima, J. 630, 645. \\
\hline 1.37 Unonopsis rufescens (Baill.) R. E. Fr. & 1 & Steward, W. C. et al. P20268. \\
\hline 1.38 Unonopsis stipitata Diels & 8 & $\begin{array}{l}\text { Cid, C. A. 7567; Cid, C. A. et al. 932, 971, 6655, 1003; Loureiro, A. et } \\
\text { al. s.n. (INPA 48115); Silva, J. A. et al. 848; Thomas, W. et al. } 5245 \text {. }\end{array}$ \\
\hline 1.39 Unonopsis sp. & 1 & Thomas, W. et al. 5269. \\
\hline 1.40 Xylopia amazonica R. E. Fr. & 1 & Silva, J. A. et al. 784. \\
\hline 1.41 Xylopia benthamii R. E. Fr. & 6 & $\begin{array}{l}\text { Cid, C. A. et al. 6654, 6733, 8184; Freitas, C. A. A. et al. 182; Loureiro, } \\
\text { A. et al. s.n. (INPA 48125); Thomas, W. et al. } 5334 \text {. }\end{array}$ \\
\hline 1.42 Xylopia emarginata Mart. & 1 & Cid, C. A. et al. 6793. \\
\hline 1.43 Xylopia aff. spruceana Benth. ex Spruce & 1 & Loureiro, A. et al. s.n. (INPA 47910). \\
\hline 1.44 Xylopia sp. & 1 & Cid, C. A. et al. 7034. \\
\hline \multicolumn{3}{|l|}{ II. Gnetaceae } \\
\hline 2.1 Gnetum leyboldii Tul. & 1 & Thomas, W. et al. 5282. \\
\hline 2.2 Gnetum sp. & 2 & Cid, C. A. et al. 6891; Lima, J. 564. \\
\hline 2.3 (Gênero não identificado). & 1 & Cid, C. A. et al. 6771. \\
\hline
\end{tabular}

espeleologia, a pesquisa arqueológica, entre outras.

Entretanto, o grande número de visitantes e a carência de um programa de educação ambiental para a preservação das espécies locais, trouxe-nos a preocupação pelo possível desaparecimento de algumas espécies, daí a proposta da elaboração de um estudo florístico para o Município colaborando assim com o turismo ecológico.

Fatores naturais como clima, relevo, tempo e materiais de origem, combinados em intensidades diferentes, formam os diversos tipos de solo observados na região. Duas unidades destacam-se entre esses tipos de solo: os podzólicos vermelho álico e/ou distrófico e, os latossolos vermelho amarelo álico e/ ou distrófico. Ocorrem, ainda; areias quartzosas, laterita hidromórfica e latossolo amarelo (Nava, et al. 1998).

O clima da região, segundo a classificação de Köppen (1948), é do tipo Amw, caracterizado por apresentar-se chuvoso, úmido e quente, com maior incidência de chuvas no período de dezembro a maio. Regionalmente, a temperatura é uniforme ao longo de todo o ano, variando entre a máxima de $38^{\circ} \mathrm{C}$ e a mínima de $20^{\circ} \mathrm{C}$. A umidade relativa do ar apresenta-se alta e uniforme durante o ano, sendo de $97 \%$ o valor médio (Nava et al. 1998).

\section{MATERIAL E MÉTODOS}

O material botânico para esta pesquisa foi coletado no Município de Presidente Figueiredo (AM), no período de 1996 a 1999 e as observações relativas às espécies foram complementadas com as contidas nas etiquetas dos espécimes adicionais, depositados no herbário do INPA. O material fértil foi processado conforme normas clássicas para este tipo de trabalho, obedecendo às recomendações do INPA com relação à incorporação das coleções básicas após identificadas, ao acervo do herbário do INPA.

Para identificação das espécies usou-se o método clássico da morfologia comparada, utilizando-se espécimes herborizados, já identificados por especialistas, comparandoos com o material ainda fresco. Para a certificação dos nomes das espécies foi utilizada a bibliografia clássica especializada usada em taxonomia, tais como: Index Kewensis (Hooker \& Jackson, 1895-1974); Gray Herbarium Index (1968-1978), Flora Brasiliensis de Martius (Martius, 1841 e Tulasne, 1863), Taxonomic Literature (Stafleu, 1967) e o Código Internacional de Nomenclatura de Botânica (Greuter et al., 2003).

\section{RESULTADOS}

Do material coletado foram identificadas 39 espécies da família Annonaceae, distribuídas em 15 gêneros, e uma espécie da família Gnetaceae, todas relacionadas na Tabela 1 .

\section{DISCUSSÃO E CONCLUSÕES}

Dentre as duas famílias estudadas para o Município de Presidente Figueiredo, a que apresentou maior diversidade foi Annonaceae, com 15 gêneros e 39 espécies. Os gêneros com maior número de espécies foram Duguetia A. St-Hill. (oito espécies), Guatteria Ruiz \& Pavon (seis espécies) e Annona L., 


\section{ACTA \\ AMAZONICA}

ESTUDOS FLORÍSTICOS NO MUNICÍPIO DE PRESIDENTE FIGUEIREDO, AMAZONAS. BRASIL - I. FAMÍLIAS ANNONACEAE E GNETACEAE
Unonopsis R. E. Fries e Xylopia L., com quatro espécies cada.

Annona impressivenia Saff. ex R. E. Fr. foi a espécie mais abundante, coletada nove vezes, seguida por Guatteria megalophylla Diels e Unonopsis stipitata Diels coletada oito vezes, Fusaea longifolia (Aubl.) Saff. coletada sete vezes, Anaxagorea brevipes Benth., Pseudoxandra coriacea R. E. Fr., Tetrameranthus laomae D. Simpson X Xylopia benthamii $\mathrm{R}$. E. Fr. coletadas seis vezes, Guatteriopsis blepharophylla (Mart.) R. E. Fr. coletada cinco vezes, Anaxagorea phaeocarpa Mart. e Duguetia stelechanta (Diels) R. E. Fr. coletadas quatro vezes. As espécies não citadas aqui foram coletadas entre uma e três vezes, conforme relacionadas na Tabela 1.

Estas famílias encontradas são comuns a outros municípios da região. Para a Flora da Reserva Ducke próximo ao Município de Manaus, Ribeiro et al. (1999) cita a maioria dos gêneros relacionados para Presidente Figueiredo. Nee (1995), também relaciona em sua Flora Preliminar, as famílias e gêneros encontrados em Figueiredo.

Com relação às espécies identificadas apenas em gêneros, não foi possível estabelecer afinidades estreitas com as espécies já identificadas no acervo do Herbário do INPA, indicando, possivelmente, serem estas espécies novas para a ciência ou novos registros para a região amazônica. Contudo, estas dúvidas somente poderão ser esclarecidas procedendo-se um estudo mais aprofundado, pelos especialistas destas famílias.

Os dados encontrados são bem satisfatórios, levando em consideração o número de amostras analisadas. Entretanto não podemos considerar que as espécies encontradas neste trabalho correspondam ao número total real para as famílias identificadas para Presidente Figueiredo. Novas coletas, certamente, levarão a um aumento no número de espécies destas famílias.

A determinação da diversidade real destas famílias só estará completa quando, por meio de sucessivas coletas, nos diferentes ecossistemas da região, não forem mais encontradas novas entidades.

\section{BIBLIOGRAFIA CITADA}

Cavalcante, P. B. 1978. Contribuição ao conhecimento das Gnetáceas da Amazônia (Gimnospermas). Acta Amazonica 8(2): 201-215.

Eletronorte/Ibama. 1996. Reserva Biológica do Uatumã - Plano de Manejo - Fase 1. Documento de Informações Básicas. Manaus. 125p.

Gray Herbarium Index. 1968/1978. Havard University. G. K. Hall \& Co. Boston. vol. 1-10, supl. 1-2.
Greuter, W.; McNeil, J.; Barrie, F. R.; Burdet, H. M.; Demoulin, V.; Filgueiras, T. S.; Nikolson, D. H.; Silva, P. C.; Skog, J. E.; Trehane, P.; Turland, N. J.; Hawksworth, D. L.. 2003. Código Internacional de Nomenclatura Botânica (Código de Saint Louis). Traduzido por C. E. M. Bicudo e J. Prado. Instituto de Botânica, São Paulo, SP. 162p.

Hooker, J. D.; Jackson, B. D. 1895/1974. Index Kewensis. Plantarum Phanerogamarum Nomina et synonima generum et specium. Clarendron Press, Oxford. vol. I-II, supl. I_XVIII.

Köppen, W. 1948. Climatologia: con un estudio de los climas de la tierra. Fondo de Cultura Econômica. México. 479p.

Markgraf, Fr. 1930. Monographie der Gattung Gnetum. Bull. Jard. Bot. Buitenz. Ser. 3, 9(4): 448-455.

Martius, C. F. Ph. von. 1841. Annonaceae. In: Martius, C. Flora Brasiliensis. 13(1):1-64. pl. 1-14.

Miralha, J. M. de S. 1995. As Annonaceae da Reserva Florestal Ducke, Manaus, AM. Manaus. INPA/UFAM. 165p. Tese de Doutorado. INPA/UFAM.

Nava, D. B.; Monteiro, E. A.; Correia, M. C.; Araújo, M. R.; Sampaio, R. R. L.; Campos, G. dos S. 1998. Companhia de Pesquisa de Recursos Minerais - CPRM. Sócio - Economia do Município de Presidente Figueiredo, Amazonas. 63p.

Nee, M. 1995. Flora Preliminar do Projeto Dinâmica Biológica de Fragmentos Florestais, New York Botanical Garden e INPA/Smithsonian Projeto Dinâmica Biológica de Fragmentos Florestais. 264p.

Ribeiro, J. E. L. do S.; Hopkins, M. J. G.; Vicentini, A.; Sothers, C. A.; Costa, M. A. da S.; Brito, J. M.; Souza, M. A. D.; Martins, L. H. P.; Lohmann, L. G.; Assunção, P. A. C. L.; Pereira, E. da C.; Silva, C. F.; Mesquita, M. R.; Procópio, L. C. 1999. Flora da Reserva Ducke: Guia de Identificação das plantas vasculares de uma floresta de terra firme na Amazônia Central. Manaus, INPA. 816p. il.

Stafleu, F. A. 1967. Taxonomic Literature. A selective guide to botanical publications with dates commentaries and types. Utrech. Regnum Vegetabili, 52: 556p.

Tulasne, L. R. 1863. Gnetaceae. In: Martius, C. F. P. von Flora Brasiliensis. 15(4): 1 pl. 398

RECEBIDO EM 21/03/2005
ACEITO EM 27/01/2006 
